\title{
Efficient selective filtering of seismic data using multiscale decomposition
}

\author{
F. E. A. Leite, M. V. C. Henriques, V. C. Gurgel, and J. M. S. Filho \\ Departamento de Ciências Exatas, Tecnológicas e Humanas, Universidade Federal Rural do Semi-Árido, Campus Angicos, \\ 59515-000, Angicos, RN, Brazil \\ Correspondence to: F. E. A. Leite (edcarlos@ufersa.edu.br)
}

Received: 17 August 2012 - Revised: 1 February 2013 - Accepted: 25 February 2013 - Published: 13 March 2013

\begin{abstract}
Seismic signal processing is an important task in geophysics sounding and represents a permanent challenge in petroleum exploration. Although seismograms could in principle give us a picture of a geological structure, they are very contaminated by spurious signals and the ground roll noise is a strongly undesired signal present in the seismograms - it does not carry physical information about the deep geological structures. This fact demands a big effort in developing new filtering methodologies. Using discrete wavelet transform, an efficient filtering for suppression of the ground roll is presented. In this method, seismic data is decomposed in multiple scales. We can remove the noise as a surgical operation in each scale, just from the regions where they are present or strong, allowing us to preserve the maximum of relevant information.
\end{abstract}

\section{Introduction}

Discrete wavelet transform is an invertible integral transformation that is implemented by measuring the correlation between the signal and one mother wavelet function. Different from the short-time fourier transform, the wavelet transform does not use a constant window to localize the events in frequency and space but uses a frequency-adaptive scale function (Daubechies, 1990). Its translations along the signal determine the location, and its stretches and squeezes determine the scale information. These properties are essential for the analysis of nonstationary signals. The scale localization corresponds to the frequency localization, due to the inverse relationship.

In geophysics the application of the discrete wavelet transform was motivated by exploration of nonstationary pro- cesses' real data which contain multiscale features by nature (Grossmann et al., 1984). The seismic data is a time series with time-varying frequencies that can be found in real processes. The study of such processes aims at transforming and representing the data so that the frequency content can be obtained locally in time. Therefore, the discrete wavelet is a convenient tool for various seismic-data-processing tasks, such as noise attenuation, because data are represented in time and frequency simultaneously (Deighan, 1997).

As a discrete transform, the discrete wavelet transform has been formulated as a unifying approach to several usages of filter banks. Daubechies, Mallat, and others demonstrated the relation between wavelet coefficient calculations, defined as integral convolutions and the filter banks (Strang te al., 1984; Mallat, 1989a,b). This joint selectivity is useful when there are regions in the signal or image whose frequency content is to be preserved or minimally affected (Corso et al., 2003; Mallat, 1992). The combination of discrete multiscale decompositions with attenuation procedures on the calculated wavelet coefficients yields filters with joint selectivity in both the time and frequency domains (Daubechies, 1990).

Figure 1a shows the graphical representation for the original seismic data analyzed. It is a set of signals (traces) acquired at several geophones (acoustic sensors) arranged at the ground level. We observe signal noise (ground roll noise in cone form) contaminating the seismic reflections (hyperboles). The ground roll is a slowly decaying, low-frequency component in seismic records, related to Rayleigh surface waves, thus appearing as a component with low constant apparent velocity (Yilmaz, 1987). As a surface component, these waves do not interact with subsurface structures associated with oil reservoirs. This means that they do not carry physical information about the deep geological structures 
and therefore they become undesirable signals. However, because of the slow decay, the ground roll appears as a strong component during a long period of time. It masks the reflective component that contains information about the deep geological structures. Because of this it is difficult to identify the reflections of interest in the regions of the seismogram where superposition of these two components occurs. Since multiscale decomposition of seismic data aims to characterize the time-dependent frequency of subsurface, the wavelet transform is well suited for such problems and has powerful application potential in seismic data processing (Deighan, 1997).

Our paper is organized as follows. In the next section we present a short mathematical background about the discrete wavelet theory of the article and fix the mathematical notation. In Sect. 3, we present in detail our method for ground roll attenuation and the results. Finally, in Sect. 4 we make some final remarks.

\section{The wavelet transform}

In this section we present the necessary information and relations concerning the continuous and discrete wavelet transforms. This mathematical framework is the basis for the decomposition of the seismic data, and it establishes the notation we use in this work. A detailed formalism of wavelet transform can be found in, among others, Daubechies (1992); Mallat (1998).

As Fourier analysis distributes a signal among a series of sines and cosines, wavelet analysis does the same with a wavelet as the decomposition basis. While the Fourier transform does not provide any spatial localization in the frequency domain, the wavelet transform is localized in frequency as well as in space. The wavelet transform doesn't use a constant window to localize the events in frequency and space as does a windowed Fourier transform but uses a frequency-adaptive scale function.

\subsection{The continuous wavelet transform}

The continuous wavelet transform $d(s, u)$ of a function $f(t) \in L^{2}(\mathbb{R})$ in the scale $s$ and time $u$ is defined as

$d(s, u)=\left\langle f, \psi_{s, u}\right\rangle=\int_{-\infty}^{+\infty} f(t) \psi_{s, u}^{\star} \mathrm{d} t$,

where

$\psi_{s, u}(t)=\frac{1}{\sqrt{|s|}} \psi\left(\frac{t-u}{s}\right)$

represents a basis of functions called wavelets. Here $\psi^{\star}$ is the complex conjugate of $\psi_{s, u}(t)$, and the parameters $s$ and $u$ are real numbers $(s \neq 0)$ that represent dilatations and translations of one fixed function, the mother wavelet $\psi(t)$.
The continuous wavelet transform is an invertible integral transformation that is implemented by measuring the correlation between the signal and one mother wavelet. Its translations along the signal determine the location, and its stretches and squeezes determine the scale information. The scale localization corresponds to the frequency localization, because scale is effectively the inverse of frequency. In continuous wavelet transform the location and scale are given by a continuous parameter.

\subsection{Discrete and orthonormal wavelet transforms}

In real situations, seismic data are discretized in time. However, in order to implement the wavelet transform on sampled signals, we need to discretize the scale and location parameters. Wavelet transforms implemented on discrete values of scale and location are called discrete wavelet transforms. A basis of orthonormal discrete wavelets can be constructed by choosing $s=2^{j}$ and $u=n 2^{j}$ with $j$ and $n$ integers. Each value of $2^{j}$ is known as scale and $j$ is the scale index. The parameter $n$ is the translation index, which determines the position of the wavelet in time. The conditions for choosing $s=2^{j}$ and $u=n 2^{j}$ are discussed by Daubechies (1992). This basis is called family Daubechies wavelets.

The corresponding discrete wavelet $\psi_{j, n}$ is defined as

$\psi_{j, n}(t)=\frac{1}{\sqrt{2^{j}}} \psi\left(\frac{t-n 2^{j}}{2^{j}}\right)$,

where $j$ and $n$, respectively, are integers associated with dilatations and translations parameters.

Let $f(t)$ be any signal in $L^{2}(\mathbb{R})$. There are bases $\left\{\psi_{j, n}\right\}$ such that $f(t)$ can be expanded, up to arbitrary high precision, by a linear combination of the wavelets as

$f(t)=\sum_{j} \sum_{n} d_{j, n} \psi_{j, n}(t)$,

where the functions $\psi_{j, n}$ are called discrete wavelets, are square integrable with zero mean, and may have some vanishing moments. The criterion of zero mean is related to the fact that one wants to have the possibility of reconstructing the original function from its wavelet decomposition. The resulting set of wavelets $\left\{\psi_{j, n}\right\}$ for all $j$ and $n$ integers form an orthogonal basis. The remarkable property of this basis is that the functions are orthogonal to their translates and their dilates (Mallat, 1989a,c; Daubechies, 1992).

The wavelet coefficients $d_{j, n}$ of the function $f(t) \in L^{2}(\mathbb{R})$ are computed by taking the inner product of $f(t)$ with the different wavelets $\psi_{j, n}$ :

$d_{j, n}=\left\langle f, \psi_{j, n}\right\rangle$.

The discrete wavelet transform of $f(t)$ is associated with timescale representation of the decomposition processes, where time location and scale are given by indices $n$ and $j$, 


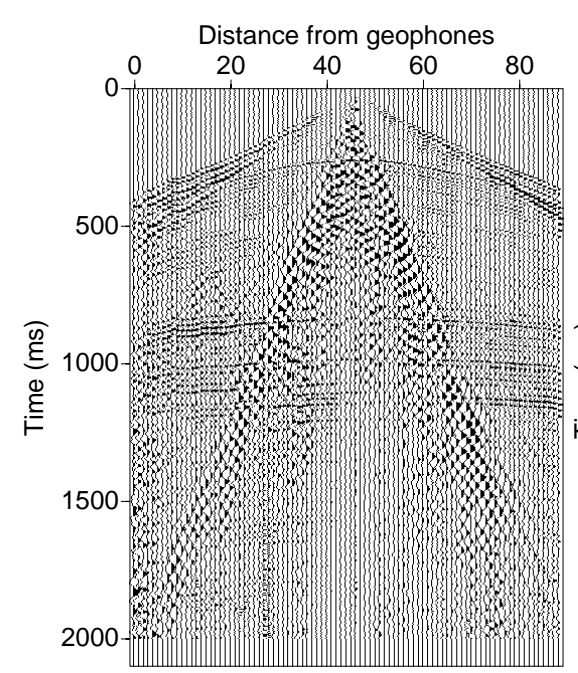

(a)

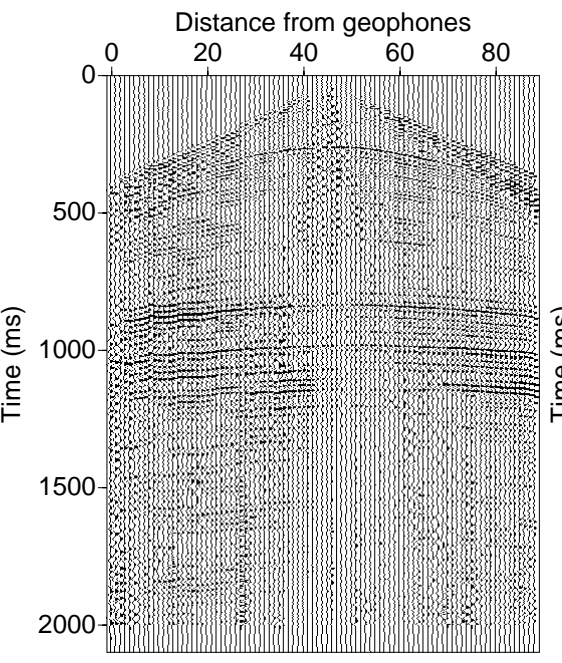

(b)

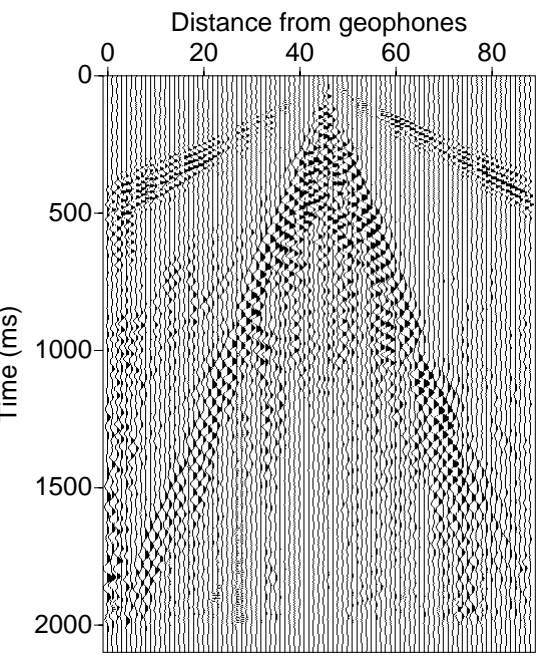

(c)

Fig. 1. (a) The original seismic data where the ground roll is present. It corresponds to record 25 as describe in Yilmaz's book. (b) This is the result after suppression of the ground roll. (c) The suppressed portion of the original seismic data.

respectively. The coefficients $d_{j, n}$ give information on the behavior of the function $f(t)$ at scale $2^{j}$ and time $2^{j} n$. Therefore representation is said to be at resolution $j$ or at scale $2^{j}$. The difference between two successive approximations is the detail at resolution $j$.

\section{Methodology and results}

We present here the analysis of seismic data which was obtained from the Center for Wave Phenomena of the Colorado School of Mines. It has 90 traces, corresponding to equally spaced geophones from the source, and 2048 samples per trace (Fig. 1a). The samples correspond to the time, both in arbitrary scale, which imply eleven scales for decomposition in the wavelet basis considered.

Our proposed method for suppressing ground roll in seismic data is based in the multiscale (multiresolution) decomposition scheme (Mallat, 1989a,c) using the orthogonal discrete wavelets. The advantages of being orthogonal are that any error in the input signal will not grow under transformation and that stable numerical computations are possible. We optimize procedure by choosing the family of Daubechies wavelets, since these wavelets are perfectly compact in time and have minimum support for a given order of approximation (Daubechies, 1988, 1992). The compactness allows a filtering process that affects strictly the events in scales and a delimited region of the seismic data. These procedures can be done in a surgical way, filtering the noise only in the spacetime region and scales in which it is present, and keeping the other regions and scales unfiltered.

In a previous filter (Corso et al., 2003), using the continuous wavelet transform, the coherent noise region was identified globally in the space-time region and the filtering was applied in a scale band below a chosen scale threshold. Considering that region of the seismic data corresponding to a bunch of low-frequency components should not be exclusively ground roll noise, an attenuation factor $A_{f}$ is introduced. When $A_{f}=0$ there is no filtering; at the opposite limit, when $A_{f}=1$ the signal is completely removed. However, we use an attenuation factor adapted for each scale. The signal reconstruction (using the inverse discrete wavelet transform) allows the partition of their components in individual scales.

The multiscale decomposition of the seismic data takes into account the timescale localization properties of the wavelet transform. The adaptative filter works in the following way. The decomposition represents the seismic data in several scales. So, for a given scale, the ground roll becomes more localized in the timescale domain and therefore can be more easily subjected to a surgical removal. In scales where the ground roll and reflections are all included, we used the attenuation factor. Thus, the filter works by reducing the coefficients, for a factor $A_{f}$, in at scale. The scales where there is just ground roll are disposed of. The attenuation factor is not used in the scales where the ground roll is not visible. Since the scale is $2^{j}$, different values of $j$ represent different scales. In this example, the index $j$ ranges from 1 to 11 . The scale index $j=1$ has the finest resolution and index $j=11$ has the coarsest resolution with the lowest frequency content. Finally we reconstruct the cleaned seismic data by scale.

The results of the decomposition in multiscale, using the discrete wavelet transform, are shown in the Fig. 2. Figures $2 \mathrm{a}$ and $\mathrm{b}$ show the seismic data for scale indexes $j=1,2$ and $j=3$, respectively. They have relatively higher frequencies which are dominated by reflection events (hyperboles). There is no significant ground roll noise is present. So, we do 


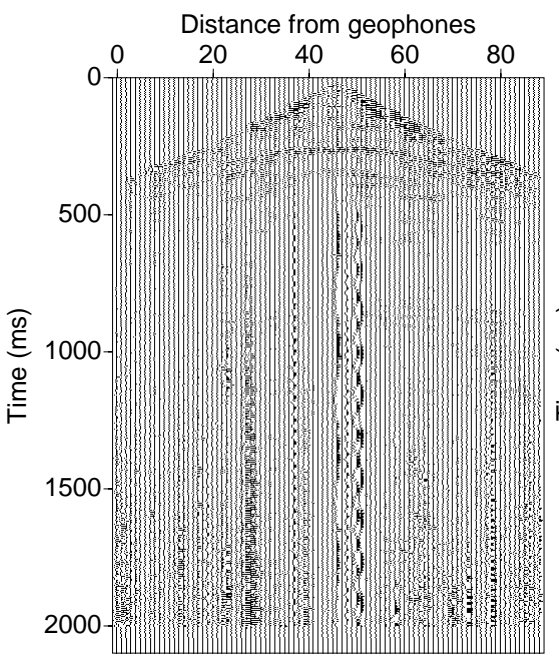

(a)

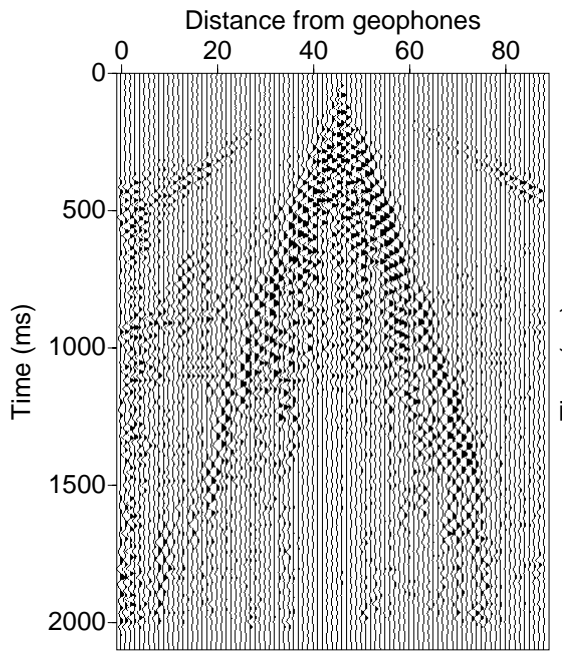

(d)

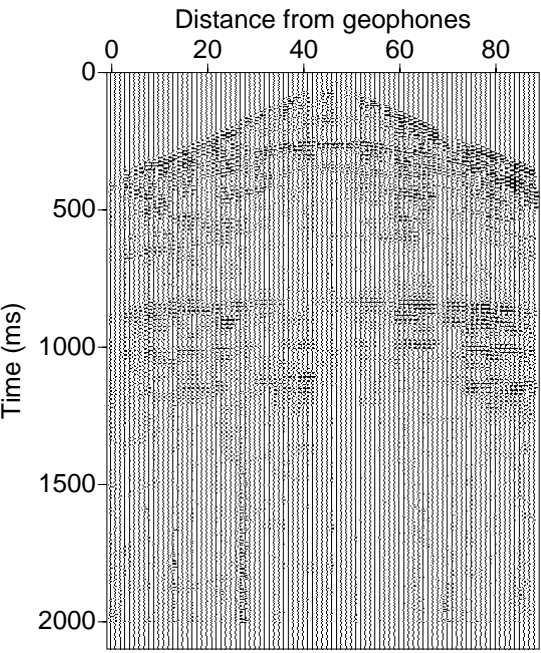

(b)

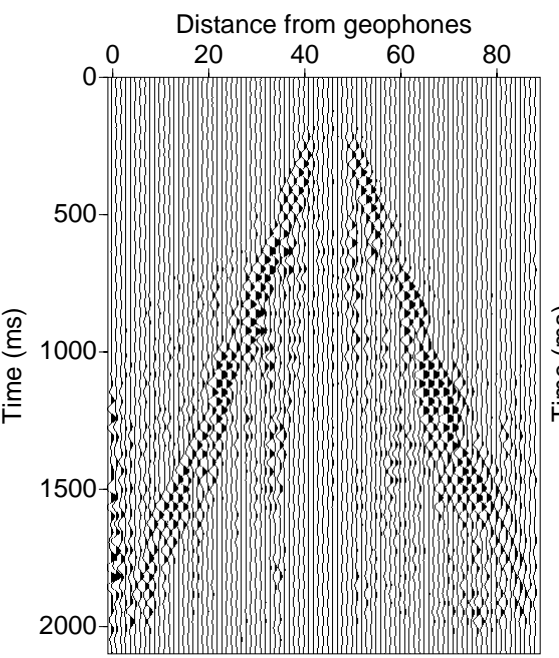

(e)

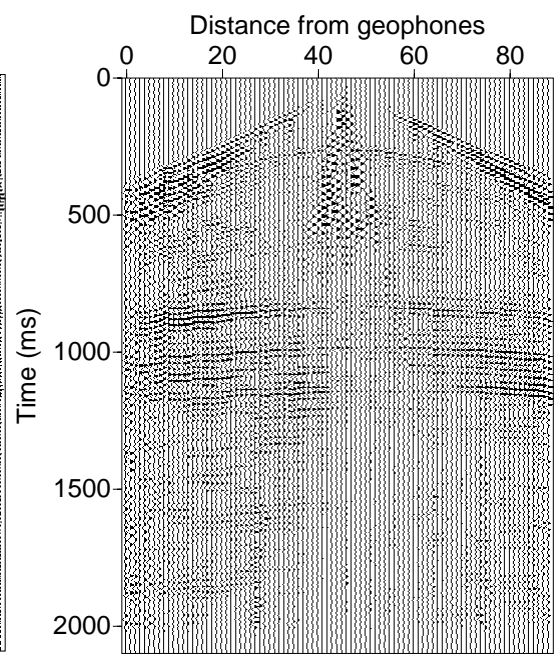

(c)

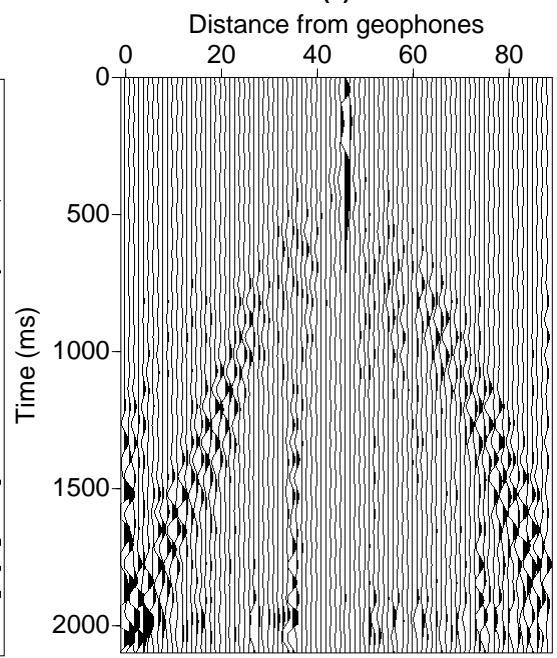

(f)

Fig. 2. Multiscale decomposition of the original seismic data (Fig. 1a), using the discrete wavelet transform. (a) is the decomposition in scales $j=1,2$ that corresponds to the largest frequency content. (b) and (c), respectively, represent decomposition in scales $j=3$ and $j=4$, which correspond to intermediate frequency. (d), (e) and (f), respectively, represent decomposition in scales $j=5, j=6$ and $j=7-11$, which correspond to the lowest frequency content.

not apply any attenuation process in these scales. In Fig. 2c we have intermediate scale index $j=4$ and we see that the ground roll is localized in a small region (in the top central region). So, we apply the filter just in this localized region of the seismic data to attenuate the ground roll. Figure $2 \mathrm{~d}$ corresponds to intermediate scale index $j=5$. In this scale the ground roll starts to appear and becomes dominant. The reflection events are almost invisible. However, a signal of physical relevance is also present. Then, we used the discrete wavelet transform following the methodology developed by Corso et al. (2003) for attenuating the ground roll in this scale. Figures $2 \mathrm{e}$ and $\mathrm{f}$ correspond to the scale indexes $j=6$ and $j=7-11$, respectively. We can notice that the seismic data just corresponds to the ground roll and no physical relevant information.
The result of the filtering process is shown in Fig. $1 \mathrm{~b}$ and c. We can note an efficient attenuation of ground roll in Fig. $1 \mathrm{~b}$. This is evidenced when one looks to Fig. 1c, where just the removed portion is shown. The reconstruction was performed by simply excluding the scales where the ground roll noise is dominant, considering the scales attenuated and the scales with just reflection events. If there is only noise in a particular scale or region, that part of the signal can be simply eliminated. As we can observe in Fig. 2c the ground roll is located in a small region. With that, it becomes easy to eliminate it without affecting the other regions. 


\section{Conclusions}

We have constructed a filter based on the discrete wavelet transform for processing seismic data. The main goal of this paper is to show an alternative method of removing the ground roll that contaminates the seismic data and disturbs their interpretation. To improve the filtering, seismic data were submitted to a multiscale decomposition. With multiscale decomposition, we have been able to localize and identify characteristics of the data in a certain scale, verifying if the ground roll is present in that scale. Then we have just attenuated it in that scale. These procedures can be done in a surgical way, filtering the noise only in the space-time region and scales in which it is present, and keeping the other regions and scales unfiltered. This operation preserves the maximum of information contained in the faint, scattered waves coming from the deep layers.

The method of multiscale decomposition has been shown to be valuable in the preservation of information we are interested in. This is particularly relevant to situations in which the characteristics of the ground roll can be well discriminated. This method shows improvements over the previous ones, especially for the high degree of adaptability to the signals, as well as for the improvement of the localization properties of the filter. In this method the filtering is much more selective and surgically precise because it is performed scale by scale. That is the reason why it is more efficient.

Acknowledgements. Financial support from the Brazilian agencies CNPq, FAPERN, FACEPE, FINEP and CTPETRO is acknowledged.

Edited by: A. Lanorte

Reviewed by: G. Corso and one anonymous referee

\section{References}

Corso, G., Kuhn, P., Lucena, L. S., and Thomé, Z.: Seismic ground roll time-frequency filtering using the gaussian wavelet transform, Physica A, 318, 551-561, 2003.

Daubechies, I.: Orthonormal bases of compactly supported wavelets, Commun. Pur Appl. Math., 41, 909-996, 1988.

Daubechies, I.: The wavelet transform, time-frequency localization and signal analysis, IEEE T. Informat. Theory, 36, 961-1005, 1990.

Daubechies, I.: Ten Lectures on wavelets, SIAM, Philadelphia, PA., 1992.

Deighan, A. J., and Watts, D. R.: Ground roll supression using the wavelet transform, Geophys., 62, 1896-1903, 1997.

Grosmann, A. and Morlet, J.: Decomposition of hardy function into square integrable wavelets of constant shape, SIAM J. Math. Anal., 15, 723-736, 1984.

Mallat, S.: A Theory for multiresolution signal decomposition: the wavelet representatio, IEEE T. Pattern Anal. Machine Intelligence, 11, 674-693, 1989.

Mallat, S.: Multifrequency channel decomposition of images and wavelet models, IEEE T. Speech and Signal Process., 37, 20912110, 1989.

Mallat, S.: Multiresolution approximations and wavelet orthonormal bases of $L^{2}(\mathbb{R})$, T. Am. Math. Soc., 315, 69-87, 1989.

Mallat, S.: Characterization of signals from multiscale edges, IEEE T. Pattern Anal. Machine Intell., 14, 710-732, 1992.

Mallat, S.: A wavelet tour of signal processing, Academic Press, 2nd ed., New York, 1998.

Strang, G. and Nguyen, T.: Wavelets and filter banks, WellesleyCambridge Press, Wellesley, USA, 1984.

Yilmaz, O.: Seismic data processing, Soc. Explor. Geophys., Tulsa, USA, 1987. 